\title{
Network Pharmacology-based Approach to Comparatively Predict the Active Ingredients and Molecular Targets of Compound Xueshuantong Capsule and Hexuemingmu Tablet in the Treatment of Proliferative Diabetic Retinopathy
}

\section{Hongyan Yao}

Ningbo Eye Hospital, Ningbo University, Ningbo 315000, People's Republic of China

\section{Danli Xin}

Ningbo Eye Hospital, Ningbo University, Ningbo 315000, People's Republic of China

\section{Zongyi Zhan}

Department of Ophthalmology, Sun Yat-sen Memorial Hospital, Sun Yat-sen University, Guangzhou 510020, People's Republic of China

\section{Zijing Li ( $\square$ lizj29@mail.sysu.edu.cn )}

Department of Ophthalmology, Sun Yat-sen Memorial Hospital, Sun Yat-sen University, Guangzhou 510020, People's Republic of China 4 Guangdong Provincial Key Laboratory of Malignant Tumor Epigenetics and Gene Regulation, Sun Yat-Sen Memorial Hospital, Sun Yat-sen University, Guangzhou 510020, People's Republic of China https://orcid.org/0000-0002-3761-6111

\section{Research}

Keywords: network, pharmacology, traditional Chinese medicine, compare, diabetic retinopathy, treatment

Posted Date: August 11th, 2020

DOI: https://doi.org/10.21203/rs.3.rs-55929/v1

License: (c) (i) This work is licensed under a Creative Commons Attribution 4.0 International License. Read Full License

Version of Record: A version of this preprint was published at Evidence-Based Complementary and Alternative Medicine on March 5th, 2021. See the published version at https://doi.org/10.1155/2021/6642600. 


\section{Abstract \\ Background}

Compound Xueshuantong capsule (CXC) and Hexuemingmu tablet (HXMMT) are two important Chinese patent medicines (CPMs) frequently used to treat proliferative diabetic retinopathy (PDR), especially when complicated with vitreous hemorrhage $(\mathrm{VH})$. However, a network pharmacology approach to understand the therapeutic mechanisms of these two CPMs in PDR has not been applied. The aim of the study was to identify differences in active ingredients between CXC and HXMMT and to comparatively predict and further analyze the molecular targets shared by these CPMs and PDR.

\section{Materials and methods}

The differentially expressed messenger RNAs (mRNAs) between normal retinal tissues in healthy individuals and active fibrovascular membranes in PDR patients were retrieved from the Gene Expression Omnibus database. The active ingredients of CXC and HXMMT and the targets of these ingredients were retrieved from the Traditional Chinese Medicine Systems Pharmacology database. The intersections of the CPM (CXC and HXMMT) targets and PDR targets were determined. Then, Gene Ontology and Kyoto Encyclopedia of Genes and Genomes (KEGG) analyses were performed, and the ingredient-target networks, protein-protein interaction networks and KEGG-target (KEGG-T) networks were constructed.

\section{Results}

There CXC contains 4 herbal drugs in CXC, and HXMMT contains 19. Radix salviae is the only herbal drug common to both. CXC had 34 potential therapeutic targets in PDR, while HXMMT had these 34 and 10 additional targets. Both CPMs shared the following main processes: response to reactive oxygen species and oxidative stress, regulation of blood vessel diameter and size, vasoconstriction, smooth muscle contraction, hemostasis and blood coagulation. The shared pathways included the AGE-RAGE signaling pathway in diabetic complications, TNF signaling pathway, relaxin signaling pathway, IL-17 signaling pathway, etc.

\section{Conclusions}

Both CXC and HXMMT include components effective in treating PDR. Radix salviae, the only herbal drug common to both CPMs, contains many useful active ingredients. HXMMT has more therapeutic targets shared by different active ingredients and more abundant gene functions than CXC. Moreover, the AGERAGE signaling pathway in diabetic complications was the most significantly enriched pathway in both networks. However, these findings should be verified through further research. 


\section{Background}

Diabetic retinopathy (DR), a serious complication of diabetes mellitus (DM) caused by microvascular ischemia and hypoxemia, affects approximately $35 \%$ of DM patients and an estimated more than 90 million people worldwide [1, 2]. The prevalence of proliferative diabetic retinopathy (PDR), a visionthreatening type of DR characterized by retinal neovascular and even vitreous hemorrhage $(\mathrm{VH})$, is nearly $7 \%$ [3]. PDR dramatically decreases patient quality of life and contributes to a massive economic burden. Therefore, it is crucial to develop effective pharmaceutical preparations to treat PDR based on its pathological mechanisms.

For thousands of years, traditional Chinese medicines (TCMs) have been used by Chinese people to treat DM and its complications [4]. Compound Xueshuantong capsule (CXC) and Hexuemingmu tablet (HXMMT) are two important Chinese patent medicines (CPMs) that are frequently used to treat PDR, especially when complicated with $\mathrm{VH}[5]$. According to observations in daily clinical practice and the results of some clinical studies, CXC and HXMMT are crucial auxiliary treatments to eliminate VH secondary to PDR as well as to improve retinal hemodynamics in PDR [4]. Correspondingly, previous experimental studies have shown that CXC and HXMMT may exert protective effects on retinal capillary endothelial cells and nerve cells by regulating multiple pathways [6-8]. However, most CMPs are composed of different kinds of herbal drugs, and every herbal drug is further composed of multiple active ingredients. Thus, a single CPM may target numerous PDR-related molecules, and the pharmacological mechanisms are complex. Moreover, in clinical practice, HXMMT more effectively eliminates new-onset VH secondary to PDR than HXMMT and is more frequently recommended by TCM doctors for new-onset VH treatment, but its potential mechanisms are poorly understood. Thus, further research is needed to better understand the underlying regulatory and interactive mechanisms of different active ingredients in CXC and HXMMT.

Network pharmacology analysis is a convenient and systematic approach to identify core targets shared by drugs and diseases [9]. It can also be performed to identify potential pathways for disease interventions, providing insight into the complex mechanisms of Chinese herbal formulas used to treat diseases. The aim of our study was to find differences in active ingredients between CXC and HXMMT and to comparatively predict and further analyze the molecular targets shared by these drugs and PDR. The flowchart of the complete procedure is shown in Fig. 1.

\section{Materials And Methods}

\subsection{Messenger RNA (mRNA) data collection and differential expression analysis}

The microarray data used in this study were retrieved from the Gene Expression Omnibus (GEO) database (http://www.ncbi.nlm.nih.gov/gds/). The mRNA expression data were acquired from dataset GSE60436, which contains 3 samples from normal retinal tissues and 3 from active fibrovascular membranes in PDR 
patients. The raw expression data were first normalized, and analysis of differentially expressed mRNAs (DEmRNAs) was then performed using the limma package based on the $\mathrm{R}$ language. The criteria for the selection of DEmRNAs were an adjusted $P$ value of $<0.05$ and a $|\log 2 \mathrm{FC}|$ value of $>1$, and the selected DEmRNAs were defined as PDR targets.

\subsection{Active ingredient screening}

A total of 499 Chinese herbal drugs and 12144 chemical ingredients from the Chinese pharmacopoeia (2010) were registered in the Traditional Chinese Medicine Systems Pharmacology (TCMSP) database (https://tcmspw.com/index.php), a platform that provides pharmacokinetic characteristics and targets of each ingredient in these herbs. Oral bioavailability (OB) and drug-likeness (DL) are two parameters commonly used to screen active ingredients. The $\mathrm{OB}$ is the percentage of the orally administered dose of the unchanged drug that enters the systemic blood circulation, and it is an important pharmacokinetic indicator. DL is used to assess whether the ingredients function as known drugs.

Each herbal drug contained in CXC and HXMMT was searched in the TCMSP database, and all ingredients were obtained. An ingredient with an $\mathrm{OB}$ of $\geq 30$ and a $\mathrm{DL}$ of $\geq 0.18$ was considered an active ingredient, and the targets of these ingredients (CXC targets and HXMMT targets) were retrieved from the database.

\subsection{Network construction}

Cytoscape 3.6.1 (http://cytoscape.org/) was used to generate all visual network diagrams, including the ingredient-target (I-T) networks, protein-protein interaction (PPI) networks and Kyoto Encyclopedia of Genes and Genomes-target (KEGG-T) networks. The intersections of the formulas' targets (CXC targets and HXMMT targets) and the PDR targets were determined. Accordingly, two I-T networks were constructed. PPI networks were constructed using the Bisogenet 3.0.0 plugin in Cytoscape 3.6.1 based on the following databases: the Database of Interacting Proteins, the Biological General Repository for Interaction Datasets, the Human Protein Reference Database, the IntAct Molecular Interaction Database, the Molecular INTeraction Database and the Binding Database. The CytoNCA plugin was used to perform topological analyses. Degree centrality and betweenness centrality were the measures selected to represent the topological features of each node in the network. Degree centrality represents the number of edges linked by a node, while betweenness centrality represents the proximity of a node to other nodes. KEGG-T networks were constructed after enrichment analyses were performed.

\subsection{Functional enrichment analysis}

Gene Ontology (GO) analyses were performed using the clusterProfiler package based on the R language. The criterion for the selection of GO processes was a $P$ value of $<0.05$. KEGG pathway analyses were performed using an online biological tool, KEGG Orthology Based Annotation System 3.0 (KOBAS 3.0, http://kobas.cbi.pku.edu.cn). The criteria for the selection of KEGG pathways were a $P$ value of $<0.01$ and a gene count of $\geq 3$. Visualizations were performed using the ggplot2 $\mathrm{R}$ package. 


\section{Results}

\subsection{Identification of PDR targets}

Analysis of the microarray dataset GSE60436 showed that 1915 mRNAs (819 upregulated mRNAs and 1096 downregulated mRNAs) were differentially expressed in PDR patients compared with individuals without PDR (Fig. 2).

\subsection{I-T network construction}

CXC contains 4 herbal drugs, and HXMMT contains 19 (Table 1). Radix salviae is the only herbal drug common to both CPMs. Through screening the active ingredients, we found 34 potential therapeutic targets in PDR for CXC (Fig. 3A) and the same 34 and 10 additional therapeutic targets in PDR for HXMMT (Fig. 3B). The ten additional targets of HXMMT were CYCS, APOD, GOT1, PECAM1, ALDH2, COL1A2, CD300A, PTGER2, CHGA and CD36. Accordingly, the active ingredients and the potential therapeutic targets were used to construct I-T networks for CXC (Fig. 4A) and HXMMT (Fig. 4B). Then, the intersections of the PDR-CXC targets and the PDR-HXMMT targets were determined, and the complete set of potential therapeutic targets of CXC was found to be included among the targets of HXMMT (Fig. 3C).

Table 1

Components of compound Xueshuantong capsule and Hexuemingmu tablet. The names in parentheses are the Chinese names of the components.

\begin{tabular}{|ll|}
\hline & Herbal drugs \\
\hline $\begin{array}{l}\text { Compound } \\
\text { Xueshuantong } \\
\text { capsule (CXC) }\end{array}$ & $\begin{array}{l}\text { Radix salviae (Danshen), Panax notoginseng (Sanqi), Hedysarum multijugum } \\
\text { Maxim. (Huangqi), Figwort root (Xuanshen) }\end{array}$ \\
\hline $\begin{array}{l}\text { Hexuemingmu } \\
\text { tablet } \\
\text { (HXMMT) }\end{array}$ & $\begin{array}{l}\text { Radix salviae (Danshen), Chuanxiong rhizoma (Chuanxiong), Radix paeoniae rubra } \\
\text { (Chishao), Gentianae radix et rhozima (Longdan), Scutellariae radix (Huangqin), }\end{array}$ \\
$\begin{array}{l}\text { Cassiae semen (Juemingzi), Chrysanthemi flos (Juhua), Ecliptae herba } \\
\text { (Mohanlian), Equiseti hiemalis herba (Muzei), Pollen typhae (Puhuang), Crataegus } \\
\text { pinnatifida Bunge (Shanzha), Prunellae spica (Xiakucao), Rehmanniae radix } \\
\text { praeparata (Dihuang), Plantaginis semen (Cheqianzi), Leonuri fructus (Chongweizi), } \\
\text { Fructus ligustri lucidi (Nvzhenzi), Curcumae radix (Yujin), Cortex moutan (Mudanpi), } \\
\text { Angelicae sinensis radix (Danggui) }\end{array}$ \\
\hline
\end{tabular}

A PPI network containing 1825 nodes and 36349 edges was constructed for the set of PDR-CXC targets, while another PPI network containing 2004 nodes and 38863 edges was constructed for the set of PDRHXMMT targets. The screening parameters were degree centrality and betweenness centrality. The thresholds were set at a degree centrality of $\geq 61$ in the first screen and a betweenness centrality of $\geq$ 600 in the second screen. After the first screen, 350 nodes and 10843 edges were included in the PDRCXC network, while 386 nodes and 11975 edges were included in the PDR-HXMMT network. After the second screen, 39 nodes and 406 edges were included in the PDR-CXC network, while 51 nodes and 628 edges were included in the PDR-HXMMT network. Almost all key genes in the PDR-CXC network were also 
included in the PDR-HXMMT network (RELA, HSPA8, HSP90AA, HSP90AB1, BRCA, EWSR1, CUL7, HNRNPU, MYC, CTNNB1, MDM2, YWHAZ, CDK2, AR, FN1, HUWE1, TP53, TUBB, EP300, GRB2, VCP, MCM2, EEF1A1, NTRK1, TRAF6, EGFR, PRKDC, SRC, HDAC5, APP, ESR1, AKT1, UBC and COPS5). In addition, RNF2, VNL, RPS27, COPS5, XP01, PARP1, RACK1, YWHAB and ITGA4 were included only in the PDRHXMMT network. The topological screening processes of the PPI networks are shown in Fig. 5.

\subsection{GO and KEGG pathway analyses}

The top 20 or all GO processes (when the number of processes was smaller than 20) in the biological process (BP), cellular component (CC) and molecular function (MF) categories were identified (Fig. 6). Both CXC and HXMMT may have therapeutic effects on PDR mainly via the following processes in the BP category: response to reactive oxygen species and oxidative stress, regulation of blood vessel diameter and size, vasoconstriction, smooth muscle contraction, hemostasis and blood coagulation. Additionally, GO terms such as positive regulation of blood circulation, negative regulation of cell adhesion, extracellular structure organization and response to lipopolysaccharide were enriched in HXMMT targets. In the CC category, the therapeutic targets of both CXC and HXMMT were associated mainly with the terms collagen-containing extracellular matrix, fibrillar collagen trimer and banded collagen fibril. Moreover, HXMMT targets were enriched in the term platelet alpha granule. In addition, CXC and HXMMT targets overlapped in GO MF terms, most of which were related to protein and enzyme binding and regulation.

After KEGG pathway analyses were performed using KOBAS 3.0, possible PDR-related pathways were selected, and visualizations were performed using the ggplot2 $\mathrm{R}$ package. All genes and pathways enriched with CXC targets were also enriched with HXMMT targets, and HXMMT had 5 additional target genes: IGFBP3, CYCS, CD36, PTGER2 and COL1A2. The pathway that was the most significantly enriched and had the highest gene ratio in both analyses was AGE-RAGE signaling pathway in diabetic complications. Other pathways in common included the TNF signaling pathway, relaxin signaling pathway, IL-17 signaling pathway, focal adhesion, etc. Additional pathways, such as neuroactive ligandreceptor interaction, chemokine signaling pathway and AMPK signaling pathway, were enriched with HXMMT targets. Then, the enriched pathways and their related target genes were used to construct KEGG-T networks for CXC and HXMMT. These results are shown in Fig. 7.

\section{Discussion}

The GO terms enriched with CXC and HXMMT targets were similar and focused mainly on the response to reactive oxygen species and oxidative stress, regulation of blood vessels and blood coagulation.

Radix salviae is an important component that is closely related to the response to reactive oxygen species and oxidative stress. In an in vitro model of hypoxia and reoxygenation, Hu's [10] study showed that Radix salviae obviously alleviated cardiomyocyte apoptosis and protected mitochondrial function and cell membrane skeleton integrity in H9c2 cells. In addition, Zhang's experiment in rodents [11] 
revealed that Danshen (Radix salviae) dripping pill inhibited apoptosis and exerted neuroprotective effects in the retinas of diabetic rats by increasing the expression of $\mathrm{Bcl}-2, \mathrm{Bcl}-2$ associated $\mathrm{X}$ and caspase-3 in diabetic rats. Moreover, according to the I-T networks of CXC and HXMMT, Radix salviae contained a greater number of active ingredients related to potential therapeutic targets in PDR than did the other herbal components, suggesting that Radix salviae may play a crucial role in PDR treatment. A randomized controlled trial (RCT) performed by Lian and colleagues [5] showed that a Radix salviaecontaining Chinese herbal product was effective in treating DR and in delaying the progression from nonPDR to PDR by reducing the area of capillary nonperfusion and degree of vascular leakage. Our study identified tanshinone as one of the most important active ingredients of Radix salviae. According to previous studies, tanshinone exerts protective effects on retinal pigment epithelium and retinal endothelial cells [12-14].

Regarding circulation-related effects, some studies $[7,15]$ have shown that CXC contributes to the attenuation of streptozotocin (STZ)-induced retinal lesions, including ameliorating increases in erythrocyte aggregation, plasma viscosity, and acellular vessel and pericyte loss, by reversing the hyperexpression of vascular endothelial growth factor (VEGF) and intercellular adhesion molecule-1 and endothelin-1 and the hypoexpression of pigment epithelium-derived factor and occludin in the retinas of STZ-induced rats. In addition, Liu's study [16] showed that different core bioactive ingredients in CXC had novel therapeutic uses in managing blood circulation. Panaxytriol and ginsenoside Rb1 were related to red blood cell aggregation, while angoroside $\mathrm{C}$ was involved in platelet aggregation.

Protocatechualdehyde was related to intrinsic clotting activity, while calycosin-7-0-beta-D-glucoside was related to extrinsic clotting activity. However, the effect of HXMMT has seldom been evaluated in DR; indeed, the only study is one by Long [6] conducted in rat models of branch retinal vein occlusion (BRVO), which indicated that HXMMT may alleviate retinal edema by regulating the expression of VEGF-a and improving microcirculation. Further studies should be performed to clarify the mechanism of HXMMT.

Utilizing a network pharmacology approach, Piao [17] found that MMP9 and IGF-1 (an IGF family member contained in Radix salviae) may be key therapeutic targets in DR. Consistent with Piao's result, we found that MMP9 was included in both the CXC and HXMMT I-T networks. Hyperglycemia may increase the activity of MMP9 and therefore provides growth space and nutrients for neovascularization by degrading the basement membrane and relaxing the cell structure [18]. In addition, IGF-2, another IGF family member, was included in our networks but is not targeted by Radix salviae. IGF is expressed in many tissues, including the retina, where it is found in cells such as retinal endothelial cells and retinal pigment epithelial cells. IGF is a crucial regulator of cell differentiation and is closely related to bloodretinal barrier breakdown and retinal neovascularization $[19,20]$. However, IGF-2 was related to 2 herbal components of CXC (Huangqi and Sanqi) and 9 herbal components of HXMMT (Cheqianzi, Chishao, Huangqin, Mohanlian, Mudanpi, Muzei, Nvzhenzi, Puhuang and Xiakucao), implying that different active ingredients may share common therapeutic targets. Combined and stronger therapeutic effects may be exerted on a therapeutic target shared by a greater number of active ingredients. HXMMT contains more components than CXC; therefore, HXMMT may have more therapeutic targets. In addition, many of the genes targeted only by HXMMT but not by CXC were related to circulation and blood coagulation. For 
instance, CYCS was shown to be involved in blood platelet formation and regulatory processes [21, 22]. APOD, a crucial component of lipoproteins that transports lipids and stabilizes the structure of lipoproteins, was found to also be closely related to angiogenesis, a critical pathophysiological process in PDR [23]. PECAM1 was suggested to play an important role in the maintenance of human vascular endothelial barrier integrity and function [24]. Similarly, our topological analysis showed that some genes targeted only by HXMMT had many other functions. For example, YWHAB may perform specific functions in rod photoreceptors [25]. RACK1 may promote the expression of VEGF in endothelial cells and subsequently facilitate angiogenesis [26]. PARP1, activated by reactive oxygen species, was proven to be involved in inflammation, cell death, and retinal disease progression [27, 28]. In summary, its stronger effects at a given dose and more numerous gene targets may be two major reasons that HXMMT is more strongly recommended than CXC by TCM doctors for treating fresh VH secondary to PDR.

These two CPMs shared many pathways in our study, and the AGE-RAGE signaling pathway in diabetic complications was the most significantly enriched pathway. RAGE is expressed in almost all retinal cells. Retinal Müller cells, the major glial cells in the retina, play a critical role in maintaining the structure and normal functions of the retina, and these cells express high levels of RAGE [29]. In addition, Zong's study [30] demonstrated that RAGE plays an essential role in retinal neurodegeneration induced by diabetes and that early induction of RAGE expression by hyperglycemia in retinal Müller cells contributes to the increased levels of proinflammatory cytokines, including VEGF (a crucial downstream growth factor in angiogenesis) and monocyte chemoattractant protein-1 (MCP-1), in vivo and in vitro. Moreover, Hirata [31] found that increased production of VEGF secondary to retinal Müller cell activation may account for neovascularization in PDR. Therefore, the AGE-RAGE signaling pathway may not only provide neuroprotection in DR but also participate in crosstalk between neuroprotection and vascular protection. The difference in the enriched genes between CXC and HXMMT was that one additional gene (COL1A2) was included among the HXMMT targets. COL1A2 has seldom been studied in DR; Zou's research [32] is the only DR study involving COL1A2 to date. Zou revealed that silencing of circular RNA COL1A2 (circCOL1A2) suppresses angiogenesis during PDR progression by regulating the miR-29b/VEGF axis, suggesting that circCOL1A2 and its related genes may be therapeutic targets in DR.

According to the pathway map, the AGE-RAGE signaling pathway in diabetic complications pathway is also closely connected with the PI3K-Akt signaling pathway and VEGF. The PI3K-Akt signaling pathway is one of the most frequently studied pathways in DR [33-35]. The proliferation, migration and invasion of retinal vascular endothelial cells, retinal pericytes, retinal pigment epithelial cells and microglial cells can be regulated through this pathway [36-39]. A series of pathophysiological processes, including oxidative stress regulation, inflammatory response regulation, angiogenesis and neuroprotective regulation, are also involved.

\section{Conclusions}

Both CXC and HXMMT include components effective in treating PDR and affect the following main processes: response to reactive oxygen species and oxidative stress, regulation of blood vessels and 
blood coagulation. Radix salviae, the only herbal drug common to both CPMs, contains many useful active ingredients. HXMMT has more therapeutic targets shared by different active ingredients and more abundant gene functions than CXC, which may be two major reasons that HXMMT is more strongly recommended than CXC as an auxiliary treatment for new-onset VH secondary to PDR. Moreover, AGERAGE signaling pathway in diabetic complications was the most significantly enriched pathway in both networks. However, further research is needed to verify these findings.

\section{Abbreviations}

CXC, compound Xueshuantong capsule; HXMMT, Hexuemingmu tablet, CPM, Chinese patent medicines; PDR, proliferative diabetic retinopathy; VH, vitreous hemorrhage; GO, Gene Ontology; KEGG, Kyoto Encyclopedia of Genes and Genomes; DR, diabetic retinopathy; DM, diabetes mellitus; mRNA, messenger RNA; DEmRNA, differentially expressed messenger RNA; I-T, ingredient-target; PPI, protein-protein interaction; KEGG-T, Kyoto Encyclopedia of Genes and Genomes-target; GEO, Gene Expression Omnibus; TCMSP, Traditional Chinese Medicine System Pharmacology; OB, Oral bioavailability; DL, drug-likeness; KOBAS, KEGG Orthology Based Annotation System; BP, biological process; CC, cellular component; MF, molecular function; STZ, streptozotocin; VEGF, vascular endothelial growth factor; MCP-1, monocyte chemoattractant protein-1.

\section{Declarations}

\section{Ethics approval and consent to participate}

Not applicable.

\section{Consent for publication}

Not applicable.

\section{Availability of data and material}

Data sharing not applicable to this article as no datasets were generated or analysed during the current study.

\section{Competing interests}

The authors declare that they have no competing interests.

\section{Funding}

This research was supported by the Natural Science Foundation of Ningbo, China (2015A610311), Ningbo Public Welfare Program (2019C50045) and Clinical Pharmacology Research Foundation of Guangdong Province (2019XQ09). 
Authors' contributions

Hongyan Yao, data analysis and manuscript drafting; Danli Xin, data collection and image editing; Zongyi Zhan, data collection; Zijing Li, data analysis, manuscript design and drafting.

\section{Acknowledgements}

Not applicable.

\section{References}

1. Suresh R, Yu H, Thoveson A, Swisher J, Apolinario M, Zhou B, Shah AR, Fish RH, Wykoff CC. Loss to Follow-Up Among Patients with Proliferative Diabetic Retinopathy in Clinical Practice. Am J Ophthalmol 2020.

2. Sun JK, Jampol LM. The Diabetic Retinopathy Clinical Research Network (DRCR.net) and Its Contributions to the Treatment of Diabetic Retinopathy. Ophthalmic Res. 2019;62(4):225-30.

3. Govindaswamy N, Gadde SG, Chidambara L, Bhanushali D, Anegondi N, Sinha Roy A. Quantitative evaluation of optical coherence tomography angiography images of diabetic retinopathy eyes before and after removal of projection artifacts. J Biophotonics. 2018;11(9):e201800003.

4. Zhang HW, Zhang H, Grant SJ, Wan X, Li G. Single herbal medicine for diabetic retinopathy. Cochrane Database Syst Rev. 2018;12:CD007939.

5. Lian F, Wu L, Tian J, Jin M, Zhou S, Zhao M, Wei L, Zheng Y, Wang Y, Zhang M, et al. The effectiveness and safety of a danshen-containing Chinese herbal medicine for diabetic retinopathy: a randomized, double-blind, placebo-controlled multicenter clinical trial. J Ethnopharmacol. $2015 ; 164: 71-7$.

6. Long P, Yan W, Liu J, Li M, Chen T, Zhang Z, An J. Therapeutic Effect of Traditional Chinese Medicine on a Rat Model of Branch Retinal Vein Occlusion. J Ophthalmol 2019, 2019:9521379.

7. Jian W, Yu S, Tang M, Duan H, Huang J. A combination of the main constituents of Fufang Xueshuantong Capsules shows protective effects against streptozotocin-induced retinal lesions in rats. J Ethnopharmacol. 2016;182:50-6.

8. Li RL, Wang JX, Chai LJ, Guo H, Wang H, Chen L, Hu LM, Wang SX. Xueshuantong for Injection (Lyophilized,) Alleviates Streptozotocin-Induced Diabetic Retinopathy in Rats. Chin J Integr Med 2020.

9. Pan L, Li Z, Wang Y, Zhang B, Liu G, Liu J. Network pharmacology and metabolomics study on the intervention of traditional Chinese medicine Huanglian Decoction in rats with type 2 diabetes mellitus. J Ethnopharmacol. 2020;258:112842.

10. Hu F, Koon CM, Chan JY, Lau KM, Fung KP. The cardioprotective effect of danshen and gegen decoction on rat hearts and cardiomyocytes with post-ischemia reperfusion injury. BMC Complement Altern Med. 2012;12:249. 
11. Zhang Q, Xiao X, Zheng J, Li M, Yu M, Ping F, Wang T, Wang X. Compound Danshen Dripping Pill Inhibits Retina Cell Apoptosis in Diabetic Rats. Frontiers in physiology. 2018;9:1501.

12. Guo ZL, Li Y, Liu XW, Wu MY, Guo Q, Yao XC, Wang YD, Wu WY. Sodium Tanshinone IIA Silate Alleviates High Glucose Induced Barrier Impairment of Human Retinal Pigment Epithelium through the Reduction of NF-kappaB Activation via the AMPK/p300 Pathway. Curr Eye Res. 2020;45(2):17783.

13. Qian S, Qian Y, Huo D, Wang S, Qian Q. Tanshinone lla protects retinal endothelial cells against mitochondrial fission induced by methylglyoxal through glyoxalase 1. Eur J Pharmacol. 2019;857:172419.

14. Fan K, Li S, Liu G, Yuan H, Ma L, Lu P. Tanshinone IIA inhibits high glucoseinduced proliferation, migration and vascularization of human retinal endothelial cells. Mol Med Rep. 2017;16(6):9023-8.

15. Duan H, Huang J, Li W, Tang M. Protective effects of fufang xueshuantong on diabetic retinopathy in rats. Evid Based Complement Alternat Med. 2013;2013:408268.

16. Liu H, Liang JP, Li PB, Peng W, Peng YY, Zhang GM, Xie CS, Long CF, Su WW. Core bioactive components promoting blood circulation in the traditional Chinese medicine compound xueshuantong capsule (CXC) based on the relevance analysis between chemical HPLC fingerprint and in vivo biological effects. PLoS One. 2014;9(11):e112675.

17. Piao CL, Luo JL, Jin, Tang C, Wang L, Lian FM, Tong XL. Utilizing network pharmacology to explore the underlying mechanism of Radix Salviae in diabetic retinopathy. Chin Med. 2019;14:58.

18. Herszenyi L, Hritz I, Pregun I, Sipos F, Juhasz M, Molnar B, Tulassay Z. Alterations of glutathione Stransferase and matrix metalloproteinase-9 expressions are early events in esophageal carcinogenesis. World J Gastroenterol. 2007;13(5):676-82.

19. Ismayilnajadteymurabadi $H$, Konukoglu D. The relationship between IGF-2, IGFBP-2, and IGFBP-3 levels in patients suffering from pre-diabetes. J Biol Regul Homeost Agents. 2018;32(1):63-8.

20. Clemmons DR, Sleevi M, Allan G, Sommer A. Effects of combined recombinant insulin-like growth factor (IGF)-I and IGF binding protein-3 in type 2 diabetic patients on glycemic control and distribution of IGF-I and IGF-II among serum binding protein complexes. J Clin Endocrinol Metab. 2007;92(7):2652-8.

21. Ong L, Morison IM, Ledgerwood EC. Megakaryocytes from CYCS mutation-associated thrombocytopenia release platelets by both proplatelet-dependent and -independent processes. $\mathrm{Br} \mathrm{J}$ Haematol. 2017;176(2):268-79.

22. Ledgerwood EC, Dunstan-Harrison C, Ong L, Morison IM. CYCS gene variants associated with thrombocytopenia. Platelets. 2019;30(5):672-4.

23. Lai CJ, Cheng HC, Lin CY, Huang SH, Chen TH, Chung CJ, Chang CH, Wang HD, Chuu CP. Activation of liver $X$ receptor suppresses angiogenesis via induction of ApoD. FASEB journal: official publication of the Federation of American Societies for Experimental Biology. 2017;31(12):5568-76.

24. Ren Q, Ren L, Ren C, Liu X, Dong C, Zhang X. Platelet endothelial cell adhesion molecule-1 (PECAM1) plays a critical role in the maintenance of human vascular endothelial barrier function. Cell Biochem 
Funct. 2015;33(8):560-5.

25. Inamdar SM, Lankford CK, Laird JG, Novbatova G, Tatro N, Whitmore SS, Scheetz TE, Baker SA. Analysis of 14-3-3 isoforms expressed in photoreceptors. Exp Eye Res. 2018;170:108-16.

26. Liu X, Zhu M, Yang X, Wang Y, Qin B, Cui C, Chen H, Sang A. Inhibition of RACK1 ameliorates choroidal neovascularization formation in vitro and in vivo. Exp Mol Pathol. 2016;100(3):451-9.

27. Liu SY, Song JY, Fan B, Wang Y, Pan YR, Che L, Sun YJ, Li GY. Resveratrol protects photoreceptors by blocking caspase- and PARP-dependent cell death pathways. Free Radic Biol Med. 2018;129:569-81.

28. Kovacs K, Vaczy A, Fekete K, Kovari P, Atlasz T, Reglodi D, Gabriel R, Gallyas F, Sumegi B. PARP Inhibitor Protects Against Chronic Hypoxia/Reoxygenation-Induced Retinal Injury by Regulation of MAPKs, HIF1a, Nrf2, and NFKB. Invest Ophthalmol Vis Sci. 2019;60(5):1478-90.

29. Fletcher EL, Phipps JA, Ward MM, Puthussery T, Wilkinson-Berka JL. Neuronal and glial cell abnormality as predictors of progression of diabetic retinopathy. Curr Pharm Design. 2007;13(26):2699-712.

30. Zong H, Ward M, Madden A, Yong PH, Limb GA, Curtis TM, Stitt AW. Hyperglycaemia-induced proinflammatory responses by retinal Muller glia are regulated by the receptor for advanced glycation end-products (RAGE). Diabetologia. 2010;53(12):2656-66.

31. Hirata C, Nakano K, Nakamura N, Kitagawa Y, Shigeta H, Hasegawa G, Ogata M, Ikeda T, Sawa H, Nakamura K, et al. Advanced glycation end products induce expression of vascular endothelial growth factor by retinal Muller cells. Biochem Biophys Res Commun. 1997;236(3):712-5.

32. Zou J, Liu K-C, Wang W-P, Xu Y. Circular RNA COL1A2 promotes angiogenesis via regulating miR29b/VEGF axis in diabetic retinopathy. Life Sciences 2020, 256.

33. Zong H, Ward M, Stitt AW. AGEs, RAGE, and diabetic retinopathy. Curr Diabetes Rep. 2011;11(4):24452.

34. Giurdanella G, Lazzara F, Caporarello N, Lupo G, Anfuso CD, Eandi CM, Leggio GM, Drago F, Bucolo C, Salomone S. Sulodexide prevents activation of the PLA2/COX-2/VEGF inflammatory pathway in human retinal endothelial cells by blocking the effect of AGE/RAGE. Biochem Pharmacol. 2017;142:145-54.

35. Chen L, Cui Y, Li B, Weng J, Wang W, Zhang S, Huang X, Guo X, Huang Q. Advanced glycation end products induce immature angiogenesis in in vivo and ex vivo mouse models. American journal of physiology Heart circulatory physiology. 2020;318(3):H519-h533.

36. Zhou L, Zhang S, Zhang L, Li F, Sun H, Feng J. MiR-199a-3p inhibits the proliferation, migration, and invasion of endothelial cells and retinal pericytes of diabetic retinopathy rats through regulating FGF7 via EGFR/PI3K/AKT pathway. Journal of receptor and signal transduction research 2020:1-13.

37. Yang X, Huo F, Liu B, Liu J, Chen T, Li J, Zhu Z, Lv B. Crocin Inhibits Oxidative Stress and Proinflammatory Response of Microglial Cells Associated with Diabetic Retinopathy Through the Activation of PI3K/Akt Signaling Pathway. Journal of molecular neuroscience: MN. 2017;61(4):5819. 
38. Ran Z, Zhang Y, Wen X, Ma J. Curcumin inhibits high glucose-induced inflammatory injury in human retinal pigment epithelial cells through the ROS-PI3K/AKT/mTOR signaling pathway. Mol Med Rep. 2019;19(2):1024-31.

39. Lu JM, Zhang ZZ, Ma X, Fang SF, Qin XH. Repression of microRNA-21 inhibits retinal vascular endothelial cell growth and angiogenesis via PTEN dependent-PI3K/Akt/VEGF signaling pathway in diabetic retinopathy. Exp Eye Res. 2020;190:107886.

\section{Figures}

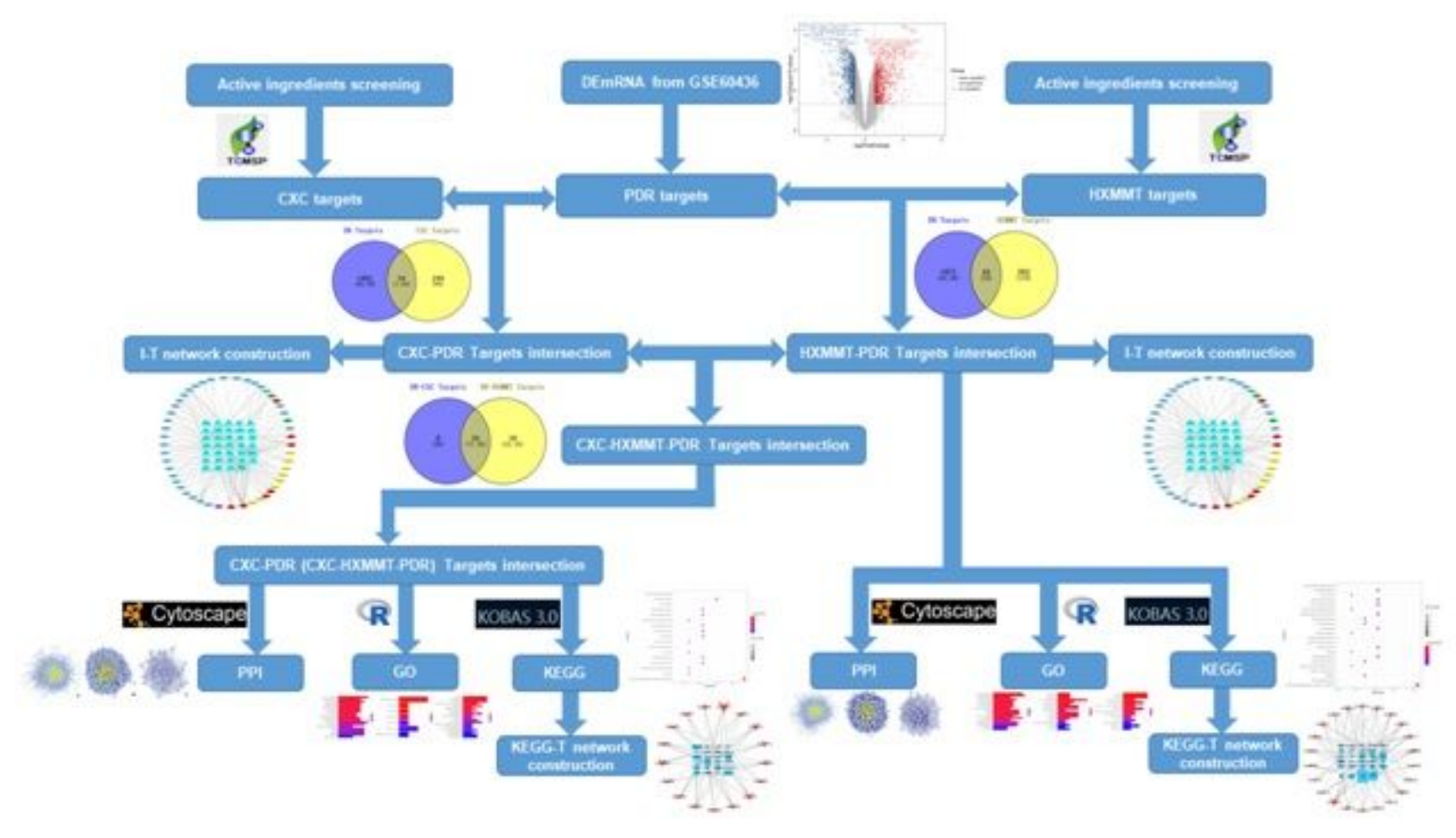

Figure 1

Flowchart of the completeanalysis procedure. DEmRNA, differentially expressed messenger RNA; CXC, compound Xueshuantong capsule; PDR, proliferative diabetic retinopathy; HXMMT,Hexuemingmu tablet; I-T, ingredient-target; PPI, protein-protein interaction; GO, Gene Ontology; KEGG, Kyoto Encyclopedia of Genes and Genomes; KEGG-T, Kyoto Encyclopedia of Genes and Genomes-target. 


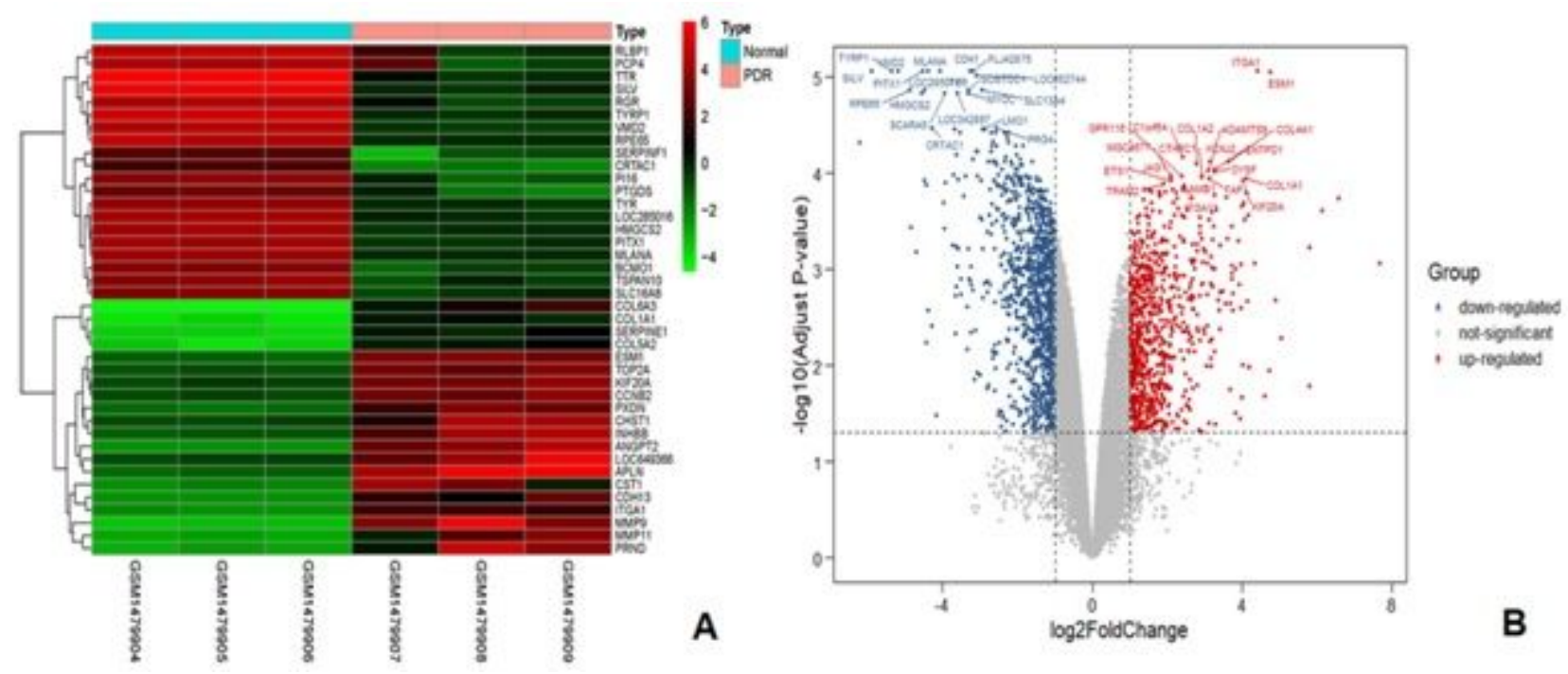

Figure 2

Heatmap (A) and volcano plot (B) of DEmRNAs (PDR targets) in microarray dataset GSE60436. The top 20 downregulated and upregulated DEmRNAs are shown in the heatmap and the volcano plot.
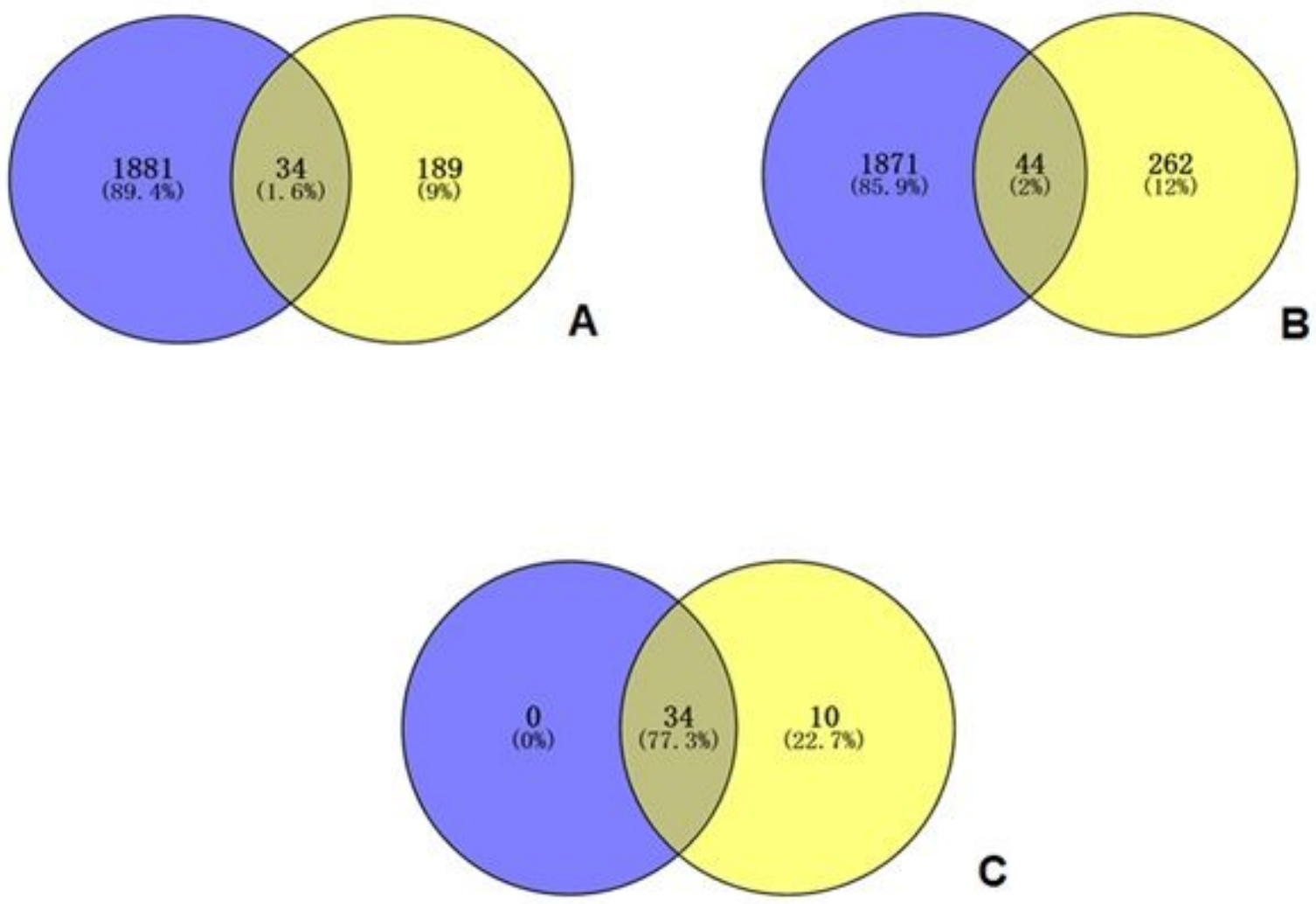

\section{Figure 3}

(A) Intersection of compound Xueshuantong capsule (CXC) targets (blue) and proliferative diabetic retinopathy (PDR) targets (yellow). (B) Intersection of Hexuemingmu tablet (HXMMT) targets (blue) and 
PDR targets (yellow). (C) Intersection of PDR-CXC targets (blue) and PDR-HXMMT targets (yellow).
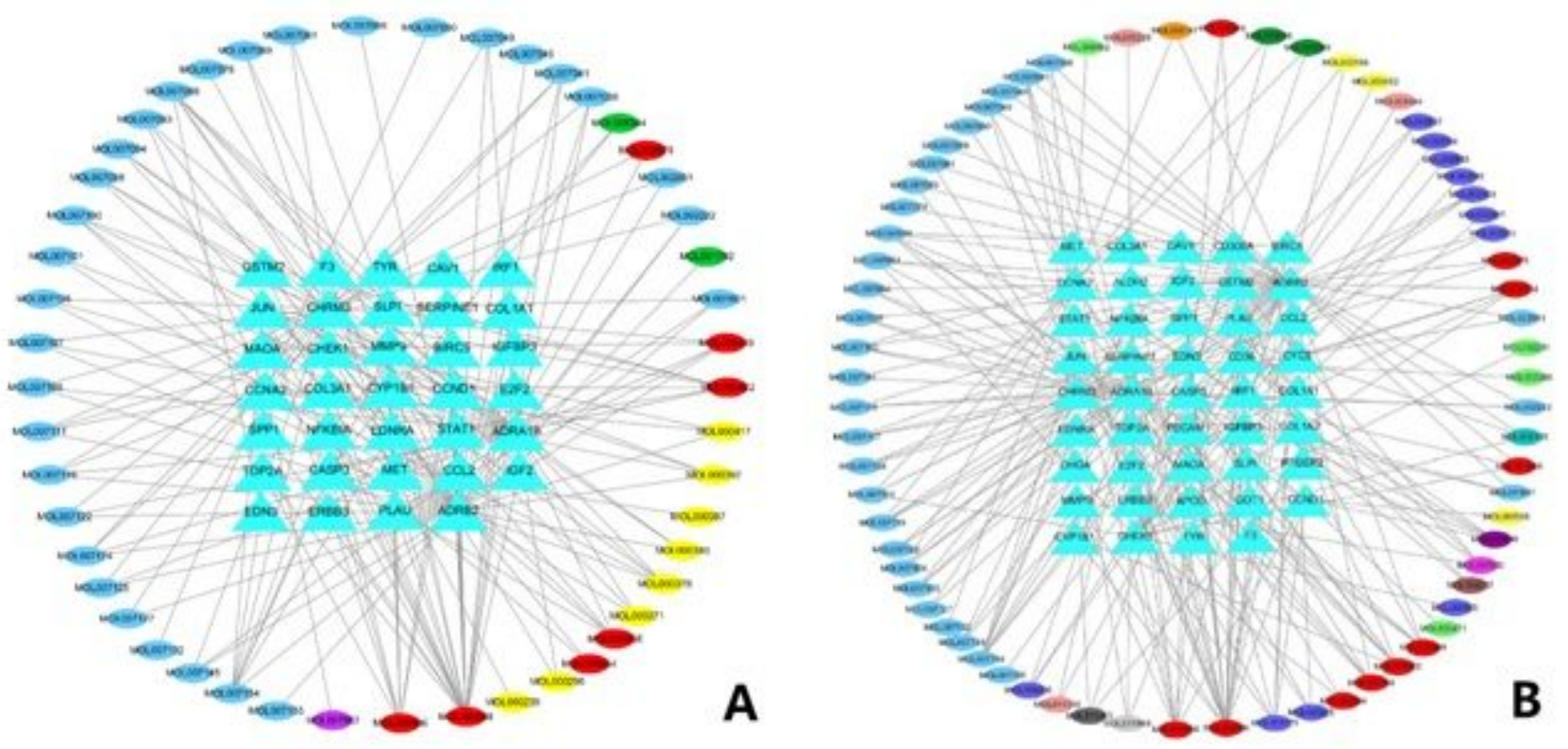

Figure 4

Ingredient-target networks ofdifferent Chinese patent medicines. The triangles and circles represent potential therapeutic targets and active ingredients, respectively. (A) Network forcompound Xueshuantong capsule.The blue circles represent active ingredients in Radix salviae, the green circles represent active ingredients in Panax notoginseng, the yellow circles represent active ingredients in HedysarummultijugumMaxim., the purple circles represent active ingredients in Figwort root, and the red circles represent active ingredients in multiple herbal drugs. (B) Network forHexuemingmu tablet.The light blue circles represent active ingredients in Radix salviae, the bluish green circles represent active ingredients in Chuanxiong rhizoma, the light purple circles represent active ingredients in Radix paeoniae rubra, the yellow circles represent active ingredients in Gentianae radix et rhozima, the dark blue circles represent active ingredients in Scutellariae radix, the light green circles represent active ingredients in Cassiae semen, the pink circles represent active ingredients in Chrysanthemiflos, the dark green circles represent active ingredients in Ecliptaeherba, the light gray circles represent active ingredients in Equisetihiemalisherba, the orange circles represent active ingredients in Fructus ligustrilucidi, the dark purple circles represent active ingredients in Pollen typhae, the dark gray circles represent active ingredients in Crataegus pinnatifida Bunge, the brown circles represent active ingredients in Prunellae spica, and the red circles represent active ingredients in multiple herbal drugs. 

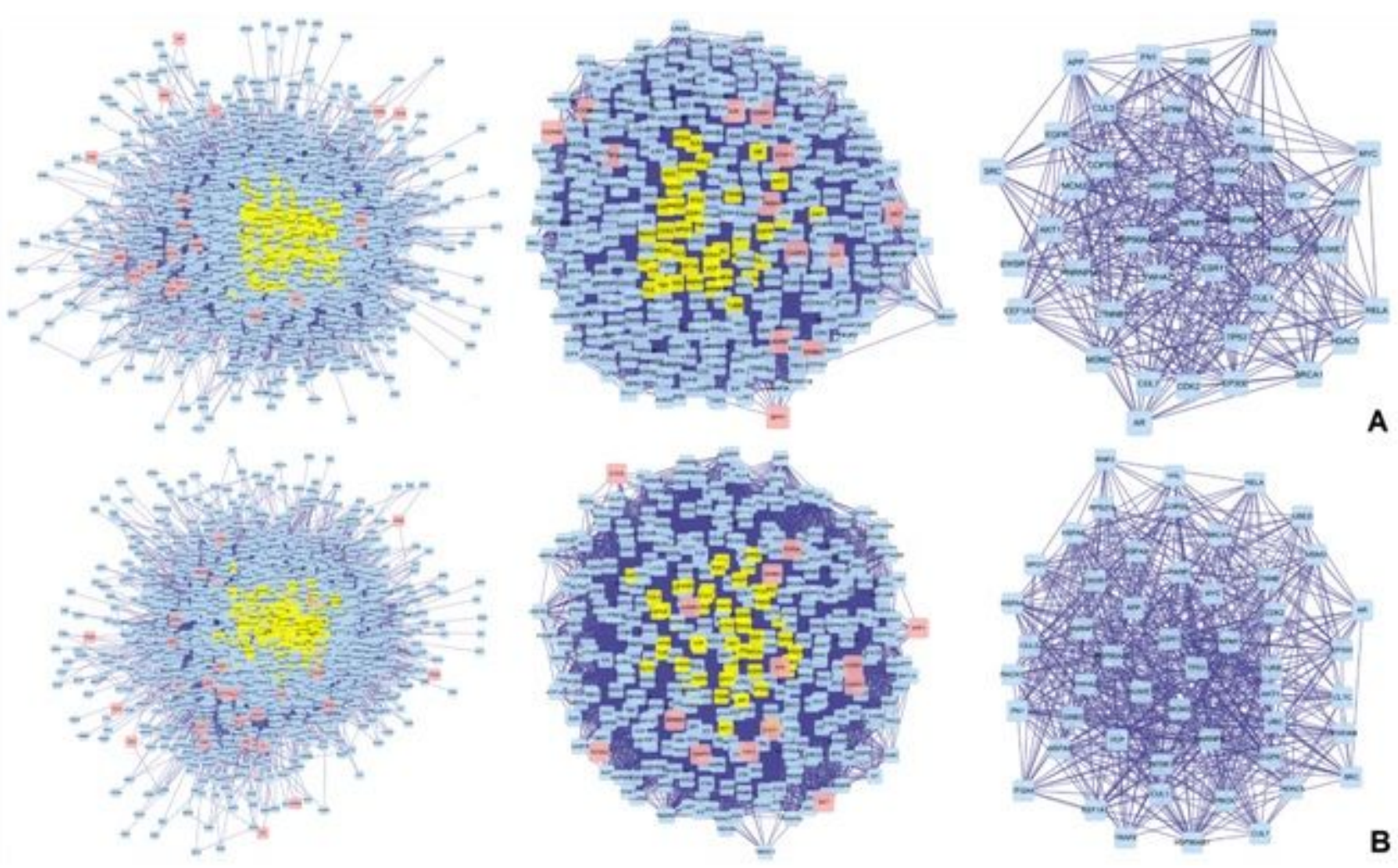

A

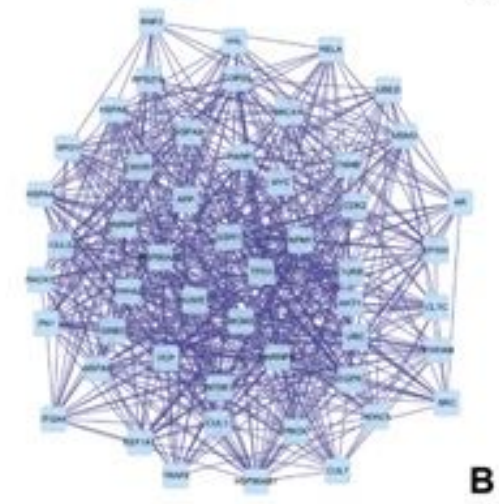

\section{Figure 5}

Topological screening process of the protein-protein interaction (PPI) networks. Left: original PPI network; middle: PPI network after the first screen; right: PPI network after the second screen. (A)Screening process of compound Xueshuantong capsule. (B) Screening process ofHexuemingmu tablet.
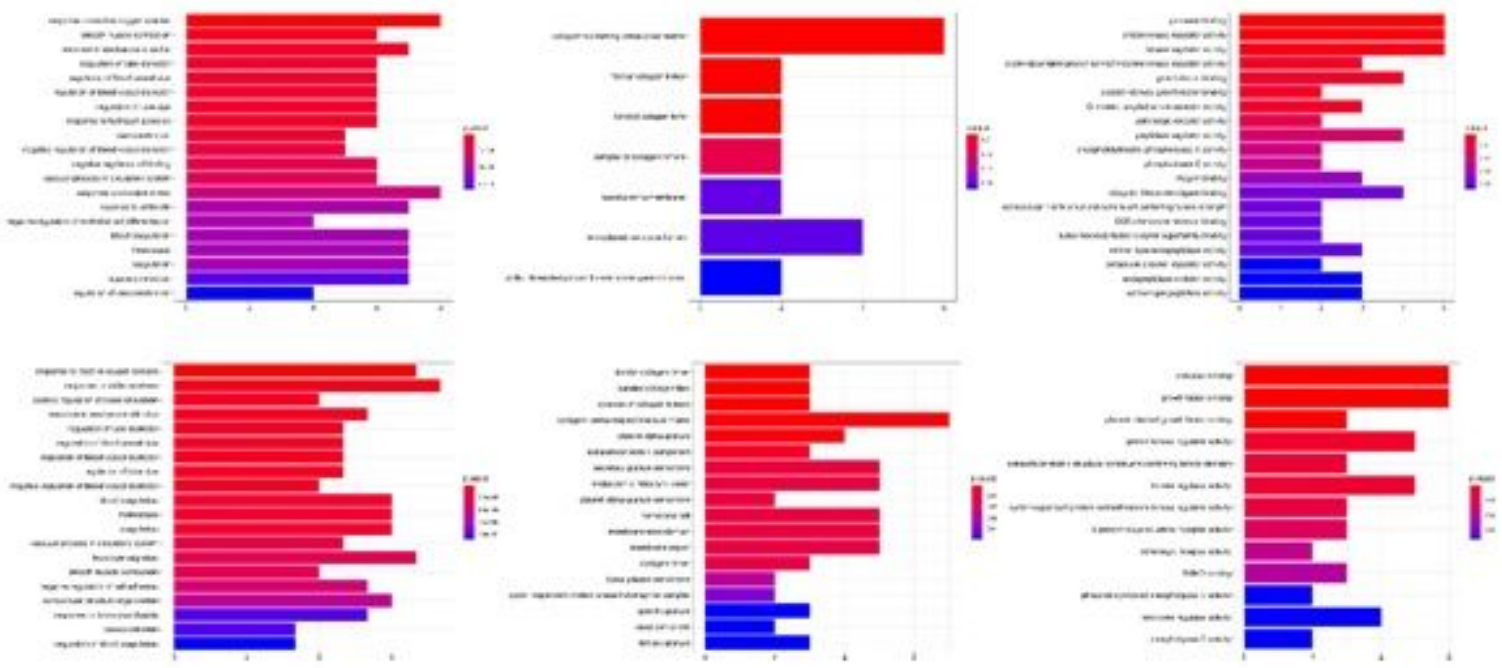

A

B

\section{Figure 6}

Gene Ontology (GO) analysis results. Left: biological process; middle: cellular component; right: molecular function. (A)GO terms enriched withtargets of compound Xueshuantong capsule. (B) GO terms enrichedwith targets of Hexuemingmu tablet. 

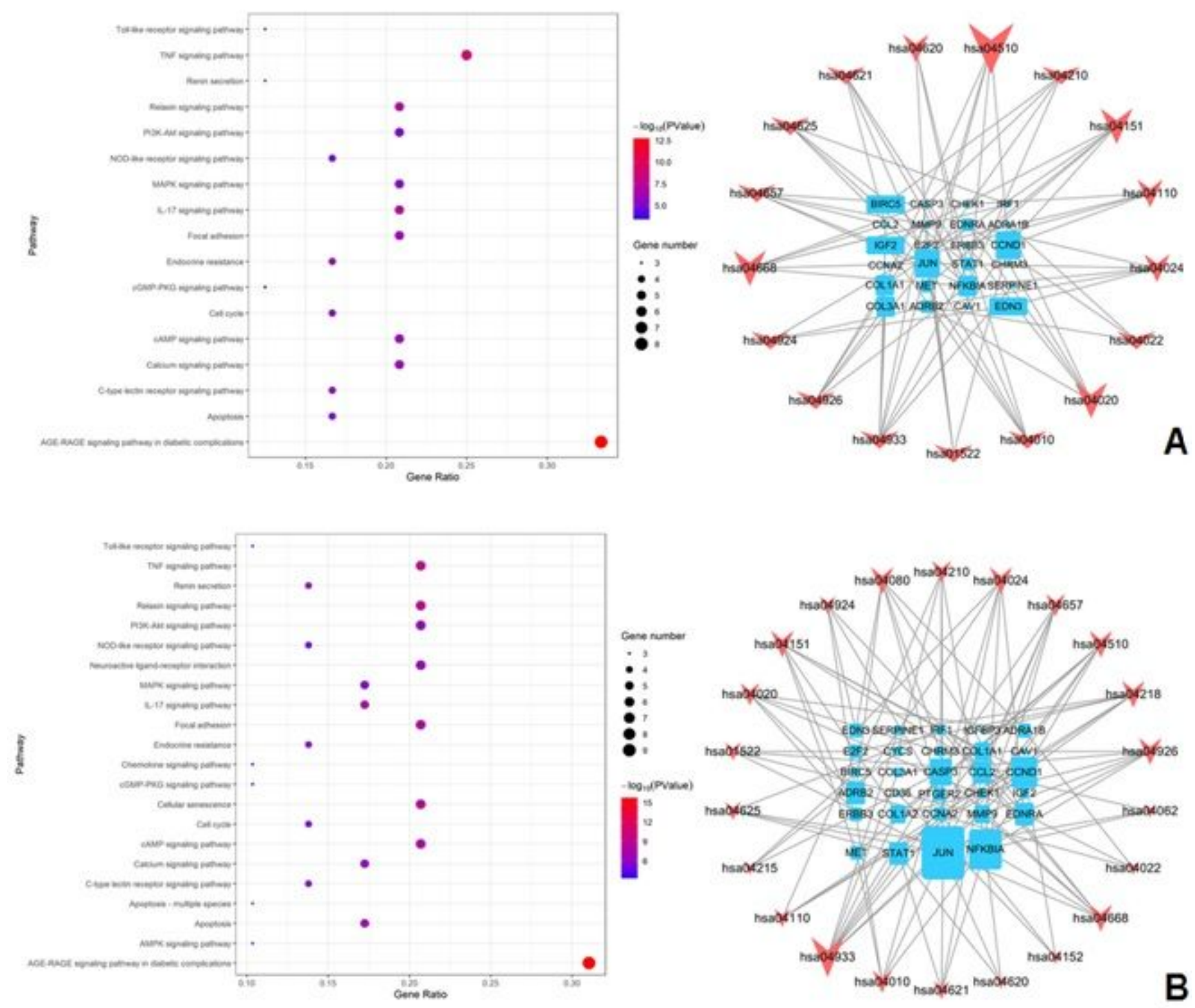

\section{Figure 7}

Kyoto Encyclopedia of Genes and Genomes (KEGG) pathway analysis results (left) and KEGG-target networks (right). In the right panel, the arrows and squares represent pathways and potential therapeutic targets, respectively. The size of the arrows represents the ratio of the corresponding therapeutic targets, while the size of the therapeutic targets represents the ratio of the corresponding pathways. (A) Pathways enriched with targets ofcompound Xueshuantong capsule. (B) Pathways enriched with targets ofHexuemingmu tablet. 


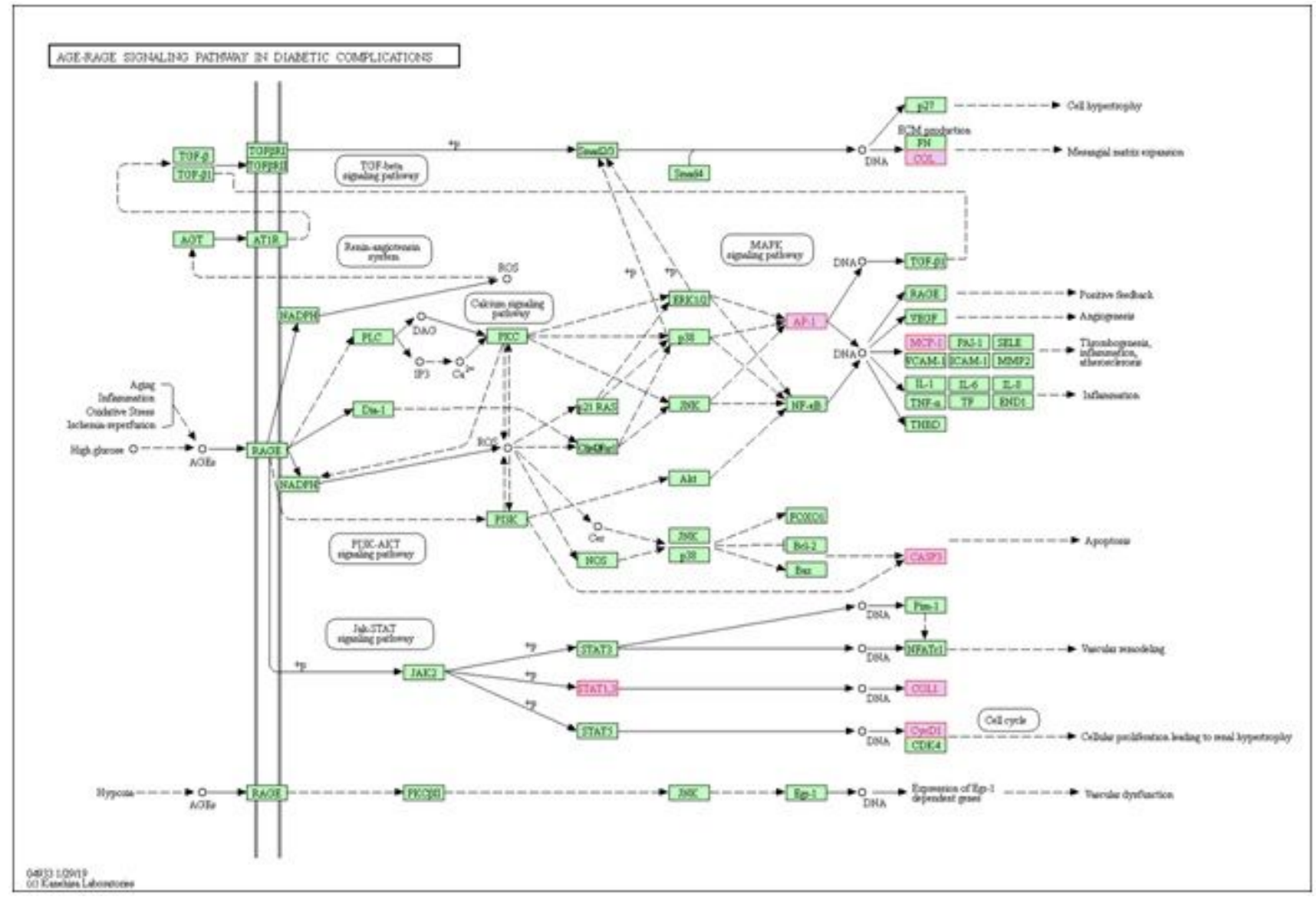

Figure 8

Most significant pathway and key genes (marked in red)amongtheproliferative diabetic retinopathy treatment targets of compound Xueshuantong capsule and Hexuemingmu tablet. 